\title{
Deciphering the trophic interaction between Akkermansia muciniphila and the butyrogenic gut commensal Anaerostipes caccae using a metatranscriptomic approach
}

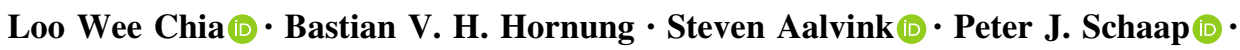 \\ Willem M. de Vos $(\mathbb{D} \cdot$ Jan Knol $\cdot$ Clara Belzer $(\mathbb{D}$
}

Received: 9 September 2017 / Accepted: 2 February 2018/Published online: 19 February 2018

(C) The Author(s) 2018. This article is an open access publication

\begin{abstract}
Host glycans are paramount in regulating the symbiotic relationship between humans and their gut bacteria. The constant flux of host-secreted mucin at the mucosal layer creates a steady niche for bacterial colonization. Mucin degradation by keystone species subsequently shapes the microbial community. This study investigated the transcriptional response during mucin-driven trophic interaction between the specialised mucin-degrader Akkermansia muciniphila and a butyrogenic gut commensal Anaerostipes caccae. A.
\end{abstract}

Electronic supplementary material The online version of this article (https://doi.org/10.1007/s10482-018-1040-x) contains supplementary material, which is available to authorized users.

L. W. Chia $\cdot$ B. V. H. Hornung $\cdot$ S. Aalvink

W. M. de Vos $\cdot$ J. Knol $\cdot$ C. Belzer $(\bowtie)$

Laboratory of Microbiology, Wageningen University \&

Research, Stippeneng 4, 6708 WE Wageningen, The

Netherlands

e-mail: clara.belzer@wur.nl

B. V. H. Hornung · P. J. Schaap

Laboratory of Systems and Synthetic Biology,

Wageningen University \& Research, Stippeneng 4,

6708 WE Wageningen, The Netherlands

W. M. de Vos

RPU Immunobiology, Faculty of Medicine, University of Helsinki, Haartmaninkatu 3, 00290 Helsinki, Finland

J. Knol

Nutricia Research, Uppsalalaan 12, 3584 CT Utrecht, The Netherlands muciniphila monocultures and co-cultures with nonmucolytic A. caccae from the Lachnospiraceae family were grown anaerobically in minimal media supplemented with mucin. We analysed for growth, metabolites (HPLC analysis), microbial composition (quantitative reverse transcription PCR), and transcriptional response (RNA-seq). Mucin degradation by $A$. muciniphila supported the growth of $A$. caccae and concomitant butyrate production predominantly via the acetyl-CoA pathway. Differential expression analysis (DESeq 2) showed the presence of $A$. caccae induced changes in the $A$. muciniphila transcriptional response with increased expression of mucin degradation genes and reduced expression of ribosomal genes. Two putative operons that encode for uncharacterised proteins and an efflux system, and several two-component systems were also differentially regulated. This indicated A. muciniphila changed its transcriptional regulation in response to A. caccae. This study provides insight to understand the mucin-driven microbial ecology using metatranscriptomics. Our findings show that the expression of mucolytic enzymes by A. muciniphila increases upon the presence of a community member. This could indicate its role as a keystone species that supports the microbial community in the mucosal environment by increasing the availability of mucin sugars.

Keywords Butyrate $\cdot$ Cross feeding $\cdot$ Keystone species $\cdot$ Microbiome $\cdot$ Mucin $\cdot$ Transcriptional regulation $\cdot$ Verrucomicrobia 


\section{Introduction}

The bacterial assembly at the mucosal layer of the human gastrointestinal tract is associated with gut health and disease (Ouwerkerk et al. 2013; Tailford et al. 2015). Although the microbial composition of the healthy mucosa has not been properly defined, it has been observed that strong deviations in the mucosal microbiota are associated with inflammatory bowel disease (IBD) (Kostic et al. 2014) and irritable bowel syndrome (IBS) (Lopez-Siles et al. 2014). At this mucosal site, host-produced mucin glycans and bioactive compounds collectively exert a selective pressure that enriches for a sub-population of mucosaassociated bacteria (Koropatkin et al. 2012; Ouwerkerk et al. 2013; Schluter and Foster 2012). Mucins are large and complex glycoproteins consisting of a protein core that is rich in proline, threonine and serine moieties, to which oligosaccharides are attached (Tailford et al. 2015). Mucins can function as an indigenous prebiotic in which only specialised members of intestinal microbiota are able to utilise it as the substrate for growth (Marcobal et al. 2013; Ouwehand et al. 2005; Tailford et al. 2015).

The intestinal symbiont, Akkermansia muciniphila is the sole human intestinal representative of the phylum Verrucomicrobia (de Vos 2017). A. muciniphila has adapted to mucosal environment in the gut (Derrien et al. 2008). The genome of A. muciniphila is equipped with an arsenal of mucin-degrading enzymes including proteases, glycosyl hydrolases $(\mathrm{GH})$, and sulfatases (Derrien et al. 2016; van Passel et al. 2011). The mucin-degrading capacity and oxygen tolerance of A. muciniphila render it a key species in the mucosal niche (Ouwerkerk et al. 2016). This specialised mucin-degrading bacterium is detected at high prevalence (over 96\%) in healthy Western adults (Collado et al. 2007; Derrien et al. 2008; Shetty et al. 2016). The abundance of A. muciniphila in the gut microbiota is inversely correlated with syndromes such as IBDs (both Crohn's disease and ulcerative colitis) (Png et al. 2010), appendicitis (Swidsinski et al. 2011) and obesity (Everard et al. 2013). Furthermore, the potential therapeutic role of $A$. muciniphila has been demonstrated in mice by remedying symptoms of obesity and diabetes (Plovier et al. 2017) as well as alcoholic liver disease (Grander et al. 2017).

In addition to the health-promoting role of $A$. muciniphila via immune modulation, the extracellular mucin degradation by this bacterium could provide growth benefits to community members via trophic interactions (Belzer et al. 2017; Belzer and de Vos 2012; Derrien et al. 2016). Several in vitro studies have demonstrated the butyrogenic effect of complex carbohydrates via cross-feeding between glycan-degrading bifidobacteria and butyrogenic bacteria (Belenguer et al. 2006; De Vuyst and Leroy 2011; Falony et al. 2006; Rios-Covian et al. 2015; Riviere et al. 2015; Schwab et al. 2017). In the mucosal environment, mucolytic bacteria such as A. muciniphila, Bacteroides spp. and Ruminococcus spp. as well as butyrogenic members of the family Lachnospiraceae (also known as Clostridium cluster XIVa) and $R u$ minococcaceae (also known as Clostridium cluster $I V$ ) are enriched (Nava et al. 2011; Van den Abbeele et al. 2013). However, no mucolytic capacities of these butyrogenic bacteria are known, which suggested potential metabolic cross-feeding between the microbial groups. Butyrate production in the vicinity of epithelial cells is suggested to be important in maintaining gut health (Koh et al. 2016; Louis and Flint 2017).

In a previous study (Belzer et al. 2017), we showed that mucin degradation by A. muciniphila yields short chain fatty acids (SCFAs) and mucin-derived monosaccharides that support the growth and concomitant butyrate production of non-mucolytic butyrogens. In this paper, we used metatranscriptomics (RNA-seq) to study the molecular response of mucindirected trophic interaction between A. muciniphila and an abutyrogenic bacterium from the family Lachnospiraceae (Anaerostipes caccae) which possesses metabolic capacity to convert acetate and lactate into butyrate (Duncan et al. 2004) and shows frequent occurrence at the mucosal niche (Nava et al. 2011; Van den Abbeele et al. 2013). We demonstrated the use of metatranscriptomics as an explorative approach to study the expressional changes of $A$. muciniphila in response to a community member. Notably, we showed that $A$. muciniphila increased its mucolytic activity to sustain the community. 


\section{Materials and methods}

Bacterial strains and growth conditions

All bacteria were grown in anaerobic serum bottles sealed with butyl-rubber stoppers at $37^{\circ} \mathrm{C}$ with $\mathrm{N}_{2}: \mathrm{CO}_{2}(80: 20$ ratio) in the headspace at $1.5 \mathrm{~atm}$. Bacterial pre-cultures were prepared by overnight growth in: minimal media supplemented with type III hog gastric mucin (Sigma-Aldrich, St. Louis, USA) for A. muciniphila $\mathrm{Muc}^{\mathrm{T}}$ (ATCC BAA-835)(Derrien et al. 2004), and peptone yeast glucose (PYG) medium for A. caccae L1-92 (DSM 14662) (Schwiertz et al. 2002). Growth was measured by spectrophotometer as optical density at $600 \mathrm{~nm}\left(\mathrm{OD}_{600}\right)$ (OD600 DiluPhotometer $^{\mathrm{TM}}$, IMPLEN, Germany).

\section{Co-culture experiment}

Co-culture experiments were performed in minimal media (Plugge 2005) supplemented with purified hog gastric mucin (Miller and Hoskins 1981). Culture conditions were established as previously described (Belzer et al. 2017). A. muciniphila was inoculated at $1 \times 10^{6}$ cells to mucin media followed by $8 \mathrm{~h}$ of incubation to allow accumulation of metabolites. Subsequently, $1 \times 10^{6}$ cells of A. caccae (A.mucA.cac co-cultures) were added to the $A$. muciniphila cultures. Cells were washed twice with phosphatebuffered saline (PBS) before addition to the cocultures to prevent carryover of metabolites from the pre-cultures. Purified mucin $\left(1.25 \mathrm{~g} \mathrm{l}^{-1}\right)$ was added to the media every $48 \mathrm{~h}$. A schematic setup of the experiment is depicted in Fig. 1a. Cultures were sampled at $0,1,2,4,6,8,11$, and 23 days for metabolites analysis. For transcriptomic analysis at day 8 , bacteria pellets were preserved in Trizol $^{\circledR}$ reagent (Invitrogen, Carlsbad, CA, USA) at $-20{ }^{\circ} \mathrm{C}$ storage till further RNA purification.

High-performance liquid chromatography (HPLC)

For metabolites analysis, $1 \mathrm{ml}$ of bacterial culture was centrifuged and the supernatant was stored at $-20{ }^{\circ} \mathrm{C}$ until HPLC analysis. Crotonate was used as the internal standard, and the external standards were lactate, formate, acetate, propionate, isobutyrate, butyrate, citrate, malate, succinate, fumarate, 1,2propanediol, methanol, ethanol, 2-propanol, lactose,
$\mathrm{N}$-acetylgalactosamine (GalNAc), $\mathrm{N}$-acetylglucosamine (GlcNAc),glucose, and galactose. Substrates conversion and products formation were measured with a Spectrasystem HPLC (Thermo Scientific, Breda, the Netherlands) equipped with a Hi-Plex-H column (Agilent, Amstelveen, the Netherlands) for the separation of organic acids and carbohydrates. A Hi-Plex-H column performs separation with diluted sulphuric acid on the basis of ionexchange ligand-exchange chromatography. Measurements were conducted at a column temperature of $45^{\circ} \mathrm{C}$ with an eluent flow of $0.8 \mathrm{ml} \mathrm{min}{ }^{-1}$ flow of $0.01 \mathrm{~N}$ sulphuric acid. Metabolites were detected by refractive index (Spectrasystem RI 150, Thermo, Breda, the Netherlands).

\section{RNA purification}

Total RNA was isolated by a method combining the Trizol $^{\circledR}$ reagent and the RNeasy Mini kit (QIAGEN $\mathrm{GmbH}$, Hilden, Germany) as described previously (Chomczynski 1993; Zoetendal et al. 2006). Four microliter of $p$-mercaptoethanol and $0.4 \mathrm{ml}$ of buffer RLT were added to $1 \mathrm{ml}$ of Trizol ${ }^{\circledR}$ reagent containing the bacterial pellet. The mixture was transferred to a tube containing $0.8 \mathrm{~g}$ of glass beads (diameter $0.1 \mathrm{~mm}$ ), followed by three times of bead beating for $1 \mathrm{~min}$ at $5.5 \mathrm{~ms}^{-1}$ with ice cooling steps in between. Subsequently, $0.2 \mathrm{ml}$ of ice-cold chloroform was added. The solution was mixed gently followed by centrifugation at $12,000 \times g$ for $15 \mathrm{~min}$ at $4{ }^{\circ} \mathrm{C}$. The RNA isolation was continued with the RNA clean-up according to the manufacturer's instructions for the RNeasy Mini kit. Genomic DNA was removed by an on-column DNase digestion step during RNA purification (DNase I recombinant, RNase-free, Roche Diagnostics GmbH, Mannheim, Germany). Yield and RNA quality was assessed using the Experion ${ }^{\mathrm{TM}}$ RNA StdSens Analysis Kit in combination with the Experion $^{\mathrm{TM}}$ System (Bio-Rad Laboratories Inc., Hercules, CA, USA).

Quantitative reverse transcription PCR (RTqPCR)

cDNA was synthesised using the ScriptSeq v2 RNASeq library preparation kit (Epicentre, Madison, WI, USA) according to the manufacturer's instructions followed by purification using CleanPCR (CleanNA, 
(a)

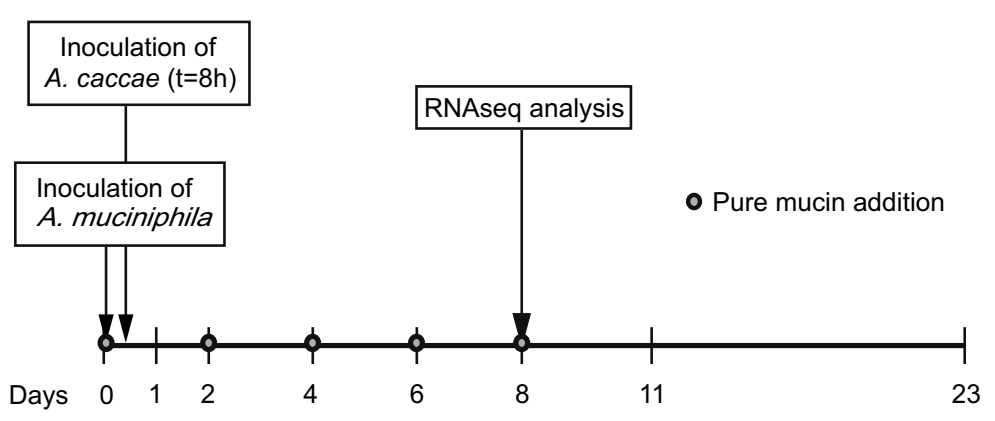

(c)

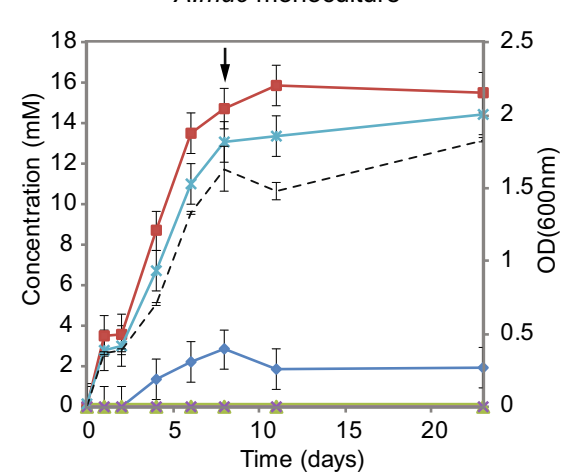

$\rightarrow$ Acetate $\rightarrow$ Succinate $\multimap$ Butyrate

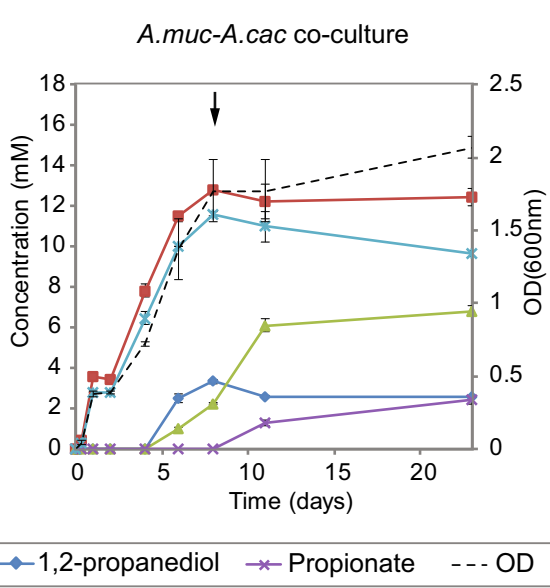

(b)

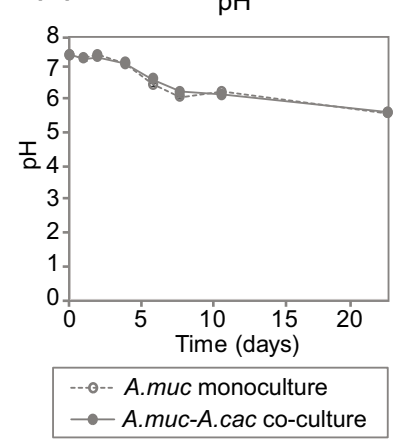

(d)

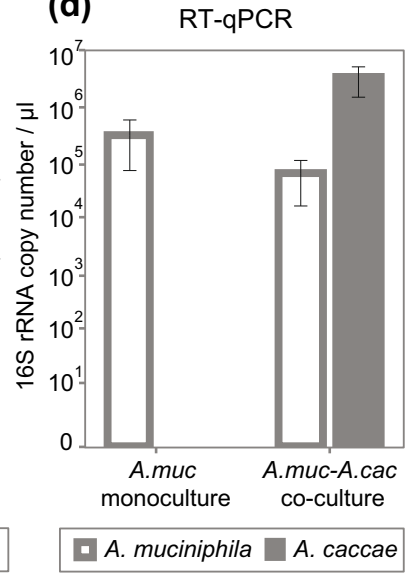

Fig. 1 a Schematic overview of the interval-fed batch culture setup. A. muciniphila was inoculated at $\mathrm{t}=0 \mathrm{~h}$ followed by $A$. caccae at $\mathrm{t}=8 \mathrm{~h}$ to ensure substrate availability for butyrogen via extracellular mucin degradation by A. muciniphila. Limited amounts of pure mucin, $0.15 \%(\mathrm{v} / \mathrm{v})$ were supplemented at 2 days intervals to maintain the abundance of $A$. muciniphila and to support the emergence of A. caccae. A sample for RNA-

the Netherlands). The cDNA was analysed by quantitative real-time PCR. Primers targeting 16S rRNA gene of A. muciniphila (AM1 5'-CAGCACGTGAAGGTGGGGAC- $3^{\prime}$ and AM2 5'-CCTTGCGGTT GGCTTCAGAT-3') (Collado et al. 2007), and $A$. caccae (OFF2555 5'-GCGTAGGTGGCATGGTAAGT- $3^{\prime}$ and OFF2556 5'-CTGCACTCCAGCATGACAGT-3') (Veiga et al. 2010) were used for quantification. Standard template DNA was prepared by $16 \mathrm{~S}$ rRNA gene amplification of each bacterium with primers 27F ( $5^{\prime}$-AGAGTTTGATCCTGGCTCAG-3') and 1492R (5'-GGTTACCTTGTTACGACTT-3'). Standard curves were prepared with nine standard concentrations from $10^{0}$ to $10^{8}$ gene copies $\mu 1^{-1}$. qPCR was performed in technical triplicate with iQ SYBR seq analysis was collected on day 8 . b The $\mathrm{pH}$ and $\mathbf{c}$ metabolite profile of monocultures and co-cultures of the interval-fed batch culture, with arrow showing day 8. d Quantification of microbial composition on day 8 by RT-qPCR targeting $16 \mathrm{~S}$ rRNA on total RNA. Error bars indicate the standard deviation of biological duplicates

Green Supermix (Bio-Rad) in a total volume of $10 \mu \mathrm{l}$ with primers at $500 \mathrm{nM}$ in 384-well plates sealed with optical sealing tape. Amplification was performed with an iCycler (Bio-Rad) with the following protocol: one cycle of $95{ }^{\circ} \mathrm{C}$ for $10 \mathrm{~min}, 35$ cycles of $95^{\circ} \mathrm{C}$ for $15 \mathrm{~s}$, $60{ }^{\circ} \mathrm{C}$ for $20 \mathrm{~s}$, and $72{ }^{\circ} \mathrm{C}$ for $30 \mathrm{~s}$ each, one cycle of $95^{\circ} \mathrm{C}$ for $1 \mathrm{~min}$, one cycle of $60^{\circ} \mathrm{C}$ for $1 \mathrm{~min}$, and a stepwise increase of the temperature from 60 to $95{ }^{\circ} \mathrm{C}$ (at $0.5{ }^{\circ} \mathrm{C}$ per $5 \mathrm{~s}$ ) to obtain melt curve data. Data were analysed using the Bio-Rad CFX Manager 3.0.

Transcriptome sequencing (RNA-seq)

Total RNA samples were further processed by Baseclear for RNA-seq (Leiden, the Netherlands). 
Depletion of ribosomal RNA was performed using the Ribo-Zero ${ }^{\mathrm{TM}}$ Kit for bacteria (Epicentre, Madison, WI, USA) followed by quality monitoring using the Agilent 2100 BioAnalyzer system. Library construction for whole transcriptome sequencing was done using the TruSeq Stranded mRNA Library Prep Kit (Illumina, USA). The barcoded cDNA libraries were analysed using BioAnalyzer and were subsequently pooled and sequenced. Single read $50 \mathrm{bp}$ sequencing was performed on two lanes using the Illumina HiSeq 2500 platform.

\section{Transcriptome analysis}

The RNA-seq data was pre-processed for quality control. Ribosomal RNA was removed with SortMeRNA v2.0 (Kopylova et al. 2012) followed by all TruSeq adapters removal with Cutadapt v1.1.a (Martin 2011). Next, quality trimming was performed using Sickle v1.33 (Joshi and Fass 2011) with a score of 30 for threshold indicating a base calling confidence of $99.9 \%$. Reads trimmed to a length $<50$ bp were removed. Reads were subsequently mapped to the relevant bacterial genomes with Bowtie2 v0.6 (Langmead and Salzberg 2012) using default settings. HTSeq v0.6.1p1 was used to determine the read count for each protein coding region (Anders et al. 2015). All these steps were performed within a local Galaxy environment (Afgan et al. 2016). More detailed information about the data analysis can be found in Table S1. Non-mapping reads of the two samples with the lowest mapping rate (both of the A. muciniphila monocultures) were collapsed to unique reads with the fastx toolkit version 0.0.14 (http://hannonlab.cshl.edu/ fastx_toolkit/). A blast search (with standard parameters, except for an e-value of 0.0001) of these unique reads was performed against the NCBI NT database (download 22.01.2014), against the human microbiome (download 08.05.2014), the NCBI bacterial draft genomes (download 23.01.2014), and the human genome (download 30.12.2013, release 08.08.2013, NCBI Homo sapiens annotation release 105). Taxonomy was estimated with a custom version of the LCA algorithm as implemented in MEGAN (Huson et al. 2011). Default parameters were used with the customization that only hits exceeding a bitscore of 50 and a length of more than 25 nucleotides were considered. $98 \%$ of the non-mapping reads were not classified, with Akkermansia accounting for $1.15 \%$ of the classified reads (Table S2). Differential gene expression was assessed using DESeq2 (Love et al. 2015). Raw RNA-seq sequence files can be accessed at the European Nucleotide Archive under accession numbers ERR1907419, ERR1907420, ERR1907423, and ERR1907424.

Carbohydrate-active enzymes (CAZymes) prediction

CAZymes were predicted with dbCAN version 3.0 (Yin et al. 2012), transmembrane domains with TMHMM version 2.0c (Krogh et al. 2001) and signal peptides with signalP 4.1 (Petersen et al. 2011).

\section{Results}

Metabolite profile of A. muciniphila monocultures and co-cultures with $A$. caccae

Co-culturing of A. muciniphila and A. caccae was performed followed by RT-qPCR, HPLC and metatranscriptomic analysis. The metabolites detected in the cultures were comparable with previous findings (Belzer et al. 2017). A. muciniphila grown as monoculture produced acetate, succinate and 1,2-propanediol as the major metabolites from pure mucin degradation (Fig. 1c). On day 8 the A.mис-A.cac cocultures yielded around $2 \mathrm{mM}$ butyrate and a low amount of propionate was detected (Fig. 1c). The mucin sugars (galactose, GalNAc, and GlcNAc) were below the detection limit of $0.5 \mathrm{mM}$.

The transcriptomes of A. muciniphila monocultures and co-cultures with $A$. caccae

Transcriptomic samples were analysed on day 8 of the interval-fed batch cultures, when the major metabolites were accumulated (Fig. 1c) and a stable bacterial composition was established (Belzer et al. 2017). On average 27 million reads were generated per sample, which is above the recommended sequence depth of 5-10 million reads for a single bacterial transcriptome (Haas et al. 2012). The detailed information about the data analysis can be found in Table S1. The RT-qPCR targeting 16S rRNA on total RNA showed an $A$. muciniphila to A. caccae ratio of 1:50 (Fig. 1d). On the other hand, the ratio of sequenced transcripts mapped 
to the genome of $A$. muciniphila versus $A$. caccae was 1:1 (Table S1).

Differential expression between A. muciniphila in monocultures and co-cultures with A. caccae

The genome of A. muciniphila possesses a total of 2176 predicted protein-coding sequences (CDSs) (van Passel et al. 2011) of which 2137 (98\%) were found to be expressed in this study (Table S3). Differential expression analysis (DESeq2) was performed to compare the gene expression of A. muciniphila in mono- and co-culture conditions. The overall transcriptional response differentiated between the monoand co-cultures (Pearson's correlation $=0.88 \pm$ 0.02) (Fig. 2).

We used cut-offs of $\mathrm{q}<0.05$ and fold change $>2$ for significantly regulated genes (Schurch et al. 2016). A total of $12 \%$ A. muciniphila genes were differentially regulated between mono- and co-cultures, with 148 upregulated genes and 132 downregulated genes (Table S3). Interestingly, two groups of contiguous genes were differentially regulated at high fold change (Fig. 3a). In the co-cultures, the upregulation of the annotated response regulator Amuc_1010 was coupled with the upregulation of a putative operon containing the genes Amuc_1011, Amuc_1012, Amuc_1013, and Amuc_1014 (Fig. 3b). Whereas, the putative operon consisting of Amuc_2041,

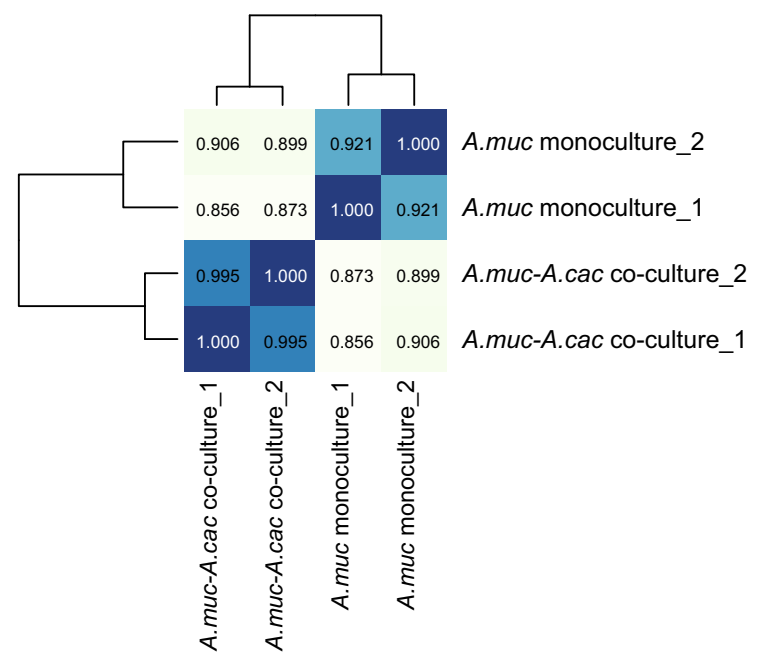

Fig. 2 Hierarchical clustering showing the Pearson's correlation of the transcriptome samples as calculated from $A$. muciniphila CDS count performed with Python 2.7.12 and SciPy version 0.17.1 (van der Walt et al. 2011)
Amuc_2042 and Amuc_2043 was downregulated in the co-cultures (Fig. 3c). Furthermore, several putative two-component systems were differentially expressed (Table 1).

Gene ontology analysis (Table 2) showed overall increase expression of hydrolase activity, DNA recombination enzymes, and sulphuric ester hydrolase activity in the co-cultures whereas ribosome, structural constituent of ribosome and translation were downregulated. The list of A. muciniphila CAZymes is summarised in Table S4. The overall expression of glycosyl hydrolases was upregulated in the co-cultures. Signal peptides and transmembrane domains prediction showed putative extracellular activity for glycosyl hydrolases required for the degradation of mucin $O$-glycan chains including GH2, GH20, GH29, GH33, GH84, GH89, and GH98.

Genes expression in relation to the metabolites production

We examined the transcripts of the co-cultures to reconcile the metabolite findings. The transcripts for A. caccae showed median of relative abundance around $0.005 \%$ and maximum value of $2.07 \%$. The list of A. caccae genes is displayed in Table S5. It is reported that $A$. caccae metabolises acetate to butyrate by employing the most prevalent butyrate production pathway via acetyl-coenzyme A (CoA) (Vital et al. 2014). The relative abundances of all transcripts involved in the metabolism pathways are summarised in Table 3. Our data indicated that the majority of enzymes involved in the acetyl-CoA pathway were expressed at a relative abundance higher than $0.1 \%$, with over $2 \%$ of total transcripts accounted for butyrate production. In addition, A. caccae possesses genomic capacity to synthesis butyrate by using 4-aminobutyrate or succinate as the precursor. However, the expression of this pathway was low, with the relative abundance of transcripts lower than $0.01 \%$, indicating that acetyl-CoA was the dominant pathway.

Nutrients interdependency between A. muciniphila and A. caccae

The genomes of A. muciniphila and A. caccae were inspected for B vitamins and amino acids auxotrophy to investigate potential nutrient interdependency. $A$. muciniphila lacked the upstream genes required for 
(a)

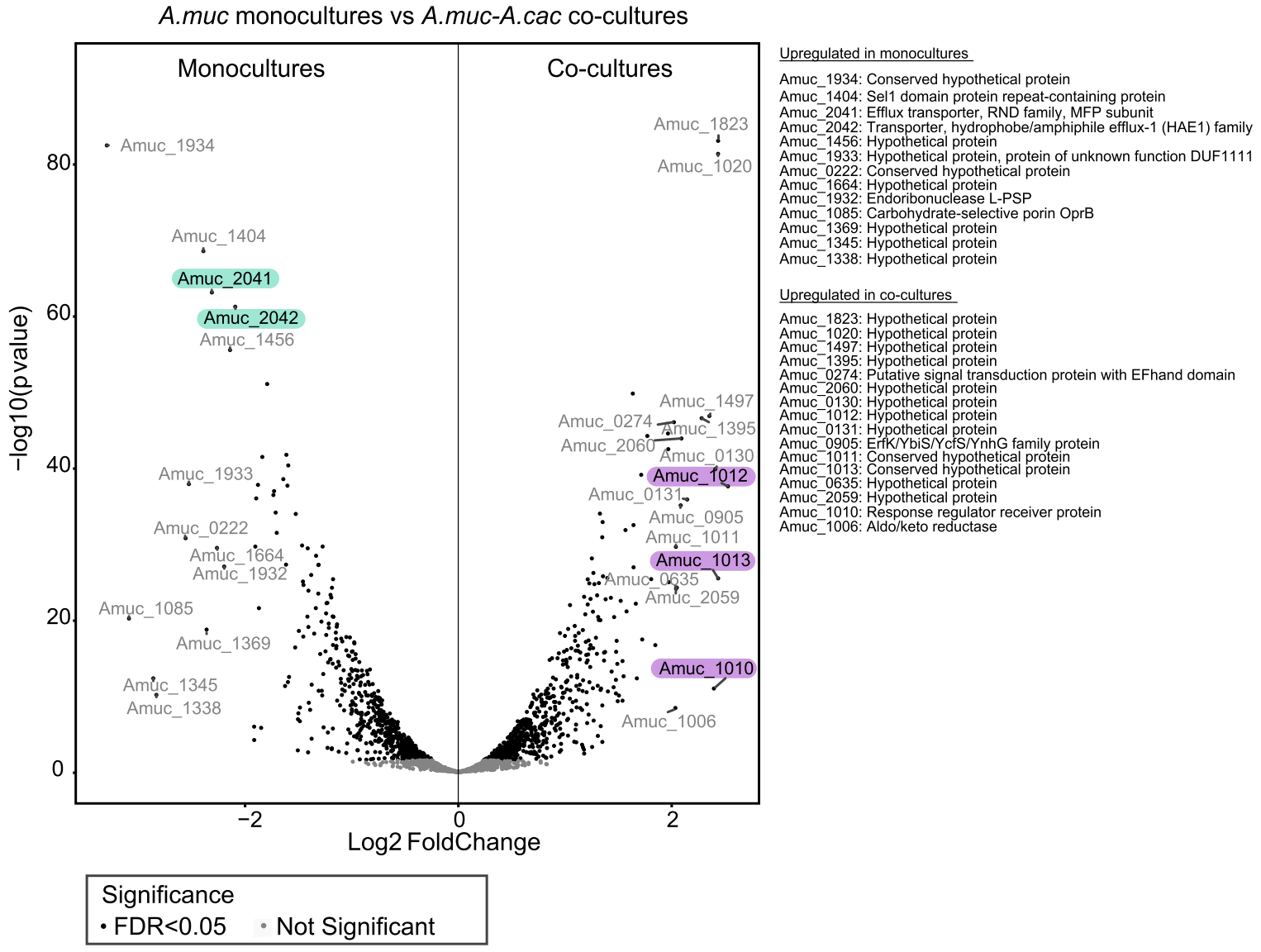

(b)

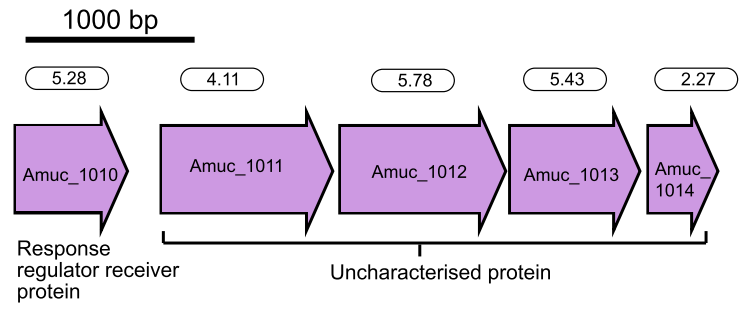

(c)

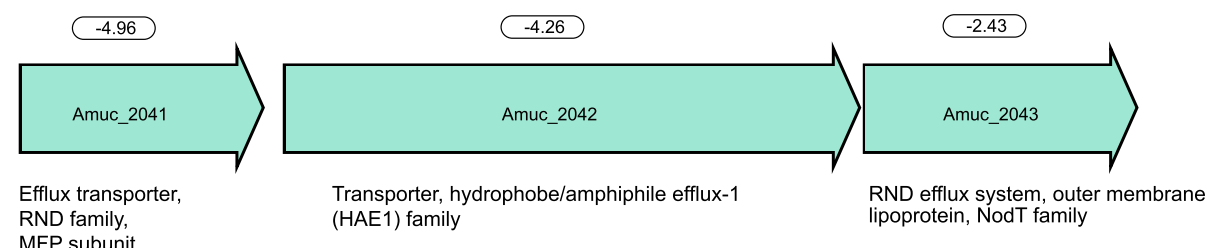

Fig. 3 a Volcano plots showing p-values correlated to fold changes in gene expression of A. muciniphila observed in monocultures versus co-cultures with A. caccae. Positive fold changes indicate upregulation in co-cultures, and negative fold changes indicate upregulation in monocultures. Locus tags for genes with $\log 2$ fold change $>2$ (or fold change $>4$ ) are labelled. $\mathbf{b}$ Response regulator and putative operon upregulated in the co-cultures. c Putative operon upregulated in the monocultures. Fold changes are listed above the respective genes 
Table 1 The differential expression of putative two-component systems in A. muciniphila

\begin{tabular}{|c|c|c|c|}
\hline \multirow[t]{2}{*}{ Locus tag } & \multicolumn{2}{|c|}{ A.тис-A.cac co-culture } & \multirow[t]{2}{*}{ Function } \\
\hline & q value & Fold change & \\
\hline Amuc_0311 & $<0.05$ & 1.96 & Signal transduction histidine kinase, nitrogenspecific, NtrB \\
\hline Amuc_0312 & $<0.05$ & 2.19 & Two-component, sigma54 specific, transcriptional regulator, Fis family \\
\hline Amuc_0827 & $<0.05$ & 1.44 & Osmo-sensitive $\mathrm{K}^{+}$channel signal transduction histidine kinase \\
\hline Amuc_0828 & $<0.05$ & 1.74 & Two-component transcriptional regulator, winged helix family \\
\hline Amuc_1109 & $<0.05$ & -1.89 & Histidine kinase \\
\hline Amuc_1110 & 0.53 & -1.07 & Two-component transcriptional regulator, winged helix family \\
\hline Amuc_1727 & 0.63 & 1.06 & Integral membrane sensor signal transduction histidine kinase \\
\hline Amuc_1728 & 0.25 & 1.13 & Two-component transcriptional regulator, winged helix family \\
\hline Amuc_1010 & $<0.05$ & 5.28 & Response regulator receiver protein \\
\hline
\end{tabular}

Negative values indicate upregulation in monocultures and positive values indicate upregulation in co-cultures

vitamin B12 biosynthesis including CbiL, CobG, CbiGF, CobF, CbiECA and CobAT. Complementarily, A. caccae was predicted to possess a complete vitamin B12 biosynthesis pathway (Table 4). However, no vitamin B12 transporter was found in the $A$. caccae genome. We found indications for aspartate auxotrophy of $A$. caccae (Table S6) however the bacterium was reported to grow in minimal defined media supplemented with glucose without additional nitrogen source (Belzer et al. 2017). Furthermore, $A$. caccae lacks the genes to synthesise the cofactor lipoate required for dehydrolipoate dehydrogenase, EC 1.8.1.4. The different enzyme complexes containing this enzyme are involved in citrate cycle, glycine, serine, and threonine metabolism, and valine, leucine, and isoleucine degradation. Nevertheless, A. caccae could acquire lipoate via salvage pathway and we observed the upregulation of lipoate biosynthesis by $A$. muciniphila in co-cultures.

\section{Discussion}

In this study, we demonstrated the use of metatranscriptomics as an explorative approach to decipher bacterial interaction in the mucosal environment. Two representative mucosa-associated species, namely $A$. muciniphila and $A$. caccae, were used to show the ecological dependency between a mucin degrader and a butyrate producer. Importantly, this study revealed changes in the expression of genes involved in hostglycan catabolism and trophic interactions between the gut commensals. This interplay leads to the formation of butyrate in the mucosal layer that is proposed to be beneficial to the host (Koh et al. 2016; Louis and Flint 2017).

In the presence of A. caccae, A. muciniphila upregulated mucin-degrading genes involved in hydrolase and sulphuric ester hydrolase activity. The majority of these mucin-degrading enzymes were predicted to function in the extracellular compartment (Ottman et al. 2016), which could lead to the degradation of oligosaccharide chains consisting of GalNAc, GlcNAc, mannose, galactose, fucose and sialic acid (Moran et al. 2011). Previous work demonstrated that A. caccae as well as Eubacterium hallii and Faecalibacterium prausnitzii could utilise the mucin-derived sugars including galactose, mannose and GlcNAc for growth (Belzer et al. 2017; Lopez-Siles et al. 2012). The fermentation of these monosaccharides results in butyrate production. Since both A. muciniphila and the butyrate-producer rely on the uptake of mucin-derived sugars for growth in our model, a higher extracellular concentration of $A$. muciniphila-derived mucolytic enzymes could contribute to substrate availability in the community. Concurrently, A. muciniphila showed downregulation of ribosomal genes in the co-cultures, which implied a lower growth rate of $A$. muciniphila. The qPCR results of genomic $16 \mathrm{~S}$ rRNA gene ratio from a previous publication on extracted DNA showed a A.muciniphila to A. caccae ratio of 100:1 (Belzer et al. 2017). In this study, the ratio of 16SrRNA in total RNA samples quantified by RT-qPCR showed a $A$. muciniphila to $A$. caccae ratio of 1:50, whereas, the sequenced transcripts ratio was 1:1. The discrepancy 
Table 2 Gene ontology (GO) analysis of the differentially regulated A. muciniphila genes $(\mathrm{q}<0.05)$ in co-cultures

\begin{tabular}{|c|c|c|c|}
\hline GO term & $\begin{array}{l}\text { Total count in A.muc } \\
\text { genome }\end{array}$ & $\begin{array}{l}\text { Percentage } \\
\text { upregulated }\end{array}$ & $\begin{array}{l}\text { Percentage } \\
\text { downregulated }\end{array}$ \\
\hline \multicolumn{4}{|l|}{ A.mис-A.cac co-culture } \\
\hline $\begin{array}{l}\text { GO:hydrolase activity, hydrolyzing O-glycosyl } \\
\text { compounds }\end{array}$ & 30 & 0.60 & $\mathbf{0 . 0 3}$ \\
\hline GO:DNA recombination & 17 & $\mathbf{0 . 5 3}$ & 0.06 \\
\hline GO:sulphuric ester hydrolase activity & 12 & 0.50 & 0.17 \\
\hline GO:transporter activity & 27 & 0.22 & 0.52 \\
\hline GO:magnesium ion binding & 16 & 0.19 & 0.44 \\
\hline GO:tRNA processing & 11 & 0.18 & 0.55 \\
\hline GO:cytoplasm & 66 & 0.17 & 0.48 \\
\hline GO:pyridoxal phosphate binding & 20 & 0.15 & 0.45 \\
\hline$G O: R N A$ binding & 37 & 0.14 & 0.46 \\
\hline GO:GTP binding & 20 & 0.10 & 0.55 \\
\hline GO:transferase activity & 21 & 0.10 & 0.43 \\
\hline GO:tRNA aminoacylation for protein translation & 24 & 0.08 & 0.71 \\
\hline GO:cellular amino acid metabolic process & 12 & 0.08 & 0.50 \\
\hline GO:aminoacyl-tRNA ligase activity & 25 & 0.08 & 0.72 \\
\hline GO:nucleotide binding & 40 & 0.08 & 0.58 \\
\hline GO:intracellular & 42 & 0.07 & 0.79 \\
\hline$G O: N A D$ binding & 15 & 0.07 & 0.33 \\
\hline GO:ribosome & 50 & 0.02 & 0.88 \\
\hline GO:structural constituent of ribosome & 55 & 0.02 & 0.89 \\
\hline GO:translation & 57 & 0.02 & 0.88 \\
\hline
\end{tabular}

The list contains GO with total count in genome higher than 10 and absolute percentage difference higher than average value. GO with overall expression upregulated or downregulated in co-cultures are marked in bold and italic respectively

could be the result of differential expression between ribosomal and messenger RNA. Note that total RNA could contain $95-99 \%$ of ribosomal RNA (Zoetendal et al. 2006) and that the number of ribosomes per cell correlates with the growth rate (Fegatella et al. 1998). In addition, $A$. muciniphila and $A$. caccae contain 3 and 12 copies of the rRNA operon, respectively. Taken together, these results indicate that $A$. muciniphila dominated in terms of cells number but $A$. caccae showed proportionally higher growth rate and transcriptional activity.

The co-culturing of two representative mucosaassociated bacteria has demonstrated the major pathways for intestinal SCFAs biosynthesis. The overview of this mucin-directed trophic interaction is shown in Fig. 4. A. caccae cross-fed on a part of the mucin sugars liberated by $A$. muciniphila for central metabolism. In addition, A. caccae can incorporate A. muciniphila-derived acetate for butyrate production via butyryl-CoA:acetate CoA-transferase enzyme(Duncan et al. 2004; Louis and Flint 2009; Louis and Flint 2017). Moreover, A. muciniphila could benefit from the corrinoids released by $A$. caccae (Degnan et al. 2014). Pseudo-vitamin B12 from $E$. hallii could activate the propionate production by $A$. muciniphila via the succinate pathway (Belzer et al. 2017). A low level of propionate was detected after day 8 in A.muc-A.cac co-cultures (Belzer et al. 2017). Propionate is likely produced by $A$. muciniphila because $A$. caccae is not known to produce propionate and it does not possess the genes involved in the known propionate biosynthesis pathways i.e. the succinate, acrylate, and propanediol pathways (Louis and Flint 2017). Nevertheless, A. caccae is predicted to synthesise vitamin B12 but lacked a vitamin B12 transporter. Upon cell lysis, the release of cellular vitamin $\mathrm{B} 12$ by $A$. caccae could facilitate methylmalonyl-CoA mutase enzymes (Amuc_1983 and 
Table 3 The relative abundance $(\%)$ of $A$. caccae transcripts for genes involved in butyrate synthesis pathway

\begin{tabular}{|c|c|c|c|}
\hline Enzyme & Locus tag & Dup1 & Dup2 \\
\hline \multicolumn{4}{|l|}{ Interconversion of pyruvate to acetyl-CoA } \\
\hline \multirow[t]{5}{*}{ Pyruvate dehyrogenase complex } & ANACAC_01488 & $<0.00$ & $<0.00$ \\
\hline & ANACAC_01489 & $<0.00$ & $<0.00$ \\
\hline & ANACAC_01490 & $<0.00$ & $<0.00$ \\
\hline & ANACAC_01491 & $<0.00$ & $<0.00$ \\
\hline & ANACAC_01492 & $<0.00$ & $<0.00$ \\
\hline \multirow[t]{2}{*}{ Formate C-acetyltransferase } & ANACAC_01621 & $<0.00$ & $<0.00$ \\
\hline & ANACAC_00664 & $<0.00$ & $<0.00$ \\
\hline Pyruvate synthase & ANACAC_00834 & 1.83 & 1.85 \\
\hline \multicolumn{4}{|l|}{ Interconversion of pyruvate to lactate } \\
\hline \multirow[t]{2}{*}{ L-Lactate dehydrogenase } & ANACAC_01148 & 0.01 & 0.01 \\
\hline & ANACAC_03769 & 0.02 & 0.02 \\
\hline \multicolumn{4}{|l|}{ Acetyl-CoA pathway } \\
\hline Acetyl-CoA C-acetyltransferase & ANACAC_00256 & 0.34 & 0.37 \\
\hline Acetoacetyl-CoA reductase & ANACAC_00254 & 0.35 & 0.39 \\
\hline \multirow[t]{2}{*}{ 3-Hydroxybutyryl-CoA dehydratase } & ANACAC_03496 & 0.01 & 0.02 \\
\hline & ANACAC_00255 & 0.21 & 0.23 \\
\hline \multirow[t]{3}{*}{ Butyryl-CoA dehydrogenase } & ANACAC_00252 & 0.50 & 0.50 \\
\hline & ANACAC_00253 & 0.54 & 0.56 \\
\hline & ANACAC_03492 & 0.00 & 0.00 \\
\hline Phosphate acetyltransferase & ANACAC_00344 & 0.13 & 0.15 \\
\hline Acetate kinase & ANACAC_00343 & 0.17 & 0.18 \\
\hline Butyryl-CoA:acetate CoA-transferase & ANACAC_01149 & 0.16 & 0.17 \\
\hline \multicolumn{4}{|l|}{ 4-Aminobutyrate/succinate pathway } \\
\hline Hydroxybutyrate dehydrogenase & ANACAC_00166 & $<0.00$ & $<0.00$ \\
\hline 4-Hydroxybutyrate coenzyme A transferase & ANACAC_00165 & $<0.00$ & $<0.00$ \\
\hline \multirow[t]{2}{*}{ 4-Hydroxybutanoyl-CoA dehydratase } & ANACAC_00167 & $<0.00$ & $<0.00$ \\
\hline & ANACAC_02698 & $<0.00$ & $<0.00$ \\
\hline
\end{tabular}

Table 4 Genomic prediction of B vitamins biosynthesis (presence $=1$ and absence $=0$ ) based on the combination of essential functional roles by Magnusdottir et al. (2015)

\begin{tabular}{|c|c|c|c|c|c|c|c|c|}
\hline & $\begin{array}{l}\text { B7 } \\
\text { Biotin }\end{array}$ & $\begin{array}{l}\text { B12 } \\
\text { Cobalamin }\end{array}$ & $\begin{array}{l}\text { B9 } \\
\text { Folate }\end{array}$ & $\begin{array}{l}\text { B3 } \\
\text { Niacin }\end{array}$ & $\begin{array}{l}\text { B5 } \\
\text { Pantothenate }\end{array}$ & $\begin{array}{l}\text { B6 } \\
\text { Pyridoxin }\end{array}$ & $\begin{array}{l}\text { B2 } \\
\text { Riboflavin }\end{array}$ & $\begin{array}{l}\text { B1 } \\
\text { Thiamin }\end{array}$ \\
\hline Akkermansia muciniphila $\mathrm{Muc}^{\mathrm{T}}$ & 1 & 0 & 1 & 1 & 1 & 1 & 1 & 1 \\
\hline Anaerostipes caccae L1-92 & 0 & 1 & 1 & 1 & 1 & 1 & 1 & 1 \\
\hline
\end{tabular}

Amuc_1984) of A. muciniphila to produce propionate (Degnan et al. 2014). The upregulation of cobalamindependent methylmalonyl-CoA mutase genes in monocultures indicated an attempt by the organism to activate the propionate production pathway in the absence of the essential cofactor (Fig. S1), as the conversion of methylmalonyl-CoA to propionyl-CoA is thermodynamically favourable (Dimroth and 
Schink 1998). The exergonic decarboxylation of methylmalonyl-CoA could be coupled to sodium ion export to extracellular space for the establishment of a proton gradient via a sodium-proton antiporter to generate ATP (Ottman et al. 2017a).

Interestingly, two putative operons and several twocomponent systems were differentially regulated, indicating the mode of transcriptional regulation by A. muciniphila in response to A. caccae. A previous study has demonstrated that the presence of one organism is often associated with transcriptional changes in the other (Plichta et al. 2016). In the coculture with A. caccae, A. muciniphila downregulated a putative operon consisting of Amuc_2041 (efflux transporter, RND family, MFP subunit), Amuc_2042 (transporter, hydrophobe/amphiphile efflux-1 (HAE1) family) and Amuc_2043 (RND efflux system, outer membrane lipoprotein, NodT family). The membrane fusion protein (MFP) is described as a component of drug resistance, nodulation, and the cell division (RND) family involved in the transportation of drug molecules (Anes et al. 2015). HAE1 is involved in toxin production and resistance processes (Anes et al. 2015). The outer membrane lipoproteins from the NodT family are predicted to primarily export small molecules rather than proteins. This efflux system was reported to play a role in multidrug resistance of Gram-negative bacteria such as Escherichia coli and Pseudomonas aeruginosa (Nikaido and Takatsuka 2009). A similar resistance mechanism could be employed by the Gram-negative A. muciniphila, and this study suggested the down-tuning of the efflux pump expression in the presence of a community member.

The annotated response regulator Amuc_1010 and the adjacent predicted operon consisting of Amuc_1011, Amuc_1012, Amuc_1013, and Amuc_1014, were upregulated in the co-cultures. Amuc_1010 is likely not a two-component system as it encoded only for the LytTR DNA-binding domain without the CheY-like receiver domain. Amuc_1010 could be autoregulatory as cis-acting regulatory elements were predicted at its upstream region using MEME (Bailey et al. 2009) (data not shown). Amuc_1011, Amuc_1012, Amuc_1013, and Amuc_1014 were annotated as uncharacterised proteins, and Amuc_1011 was predicted as an outer membrane protein (Ottman et al. 2016). Further research is needed to investigate this interesting gene cluster with unidirectional arrangement and a short intercistronic region that could likely be co-transcribed. The upregulation of the outer membrane protein could be associated with host colonization, persistence and immunomodulation (Galdiero et al. 2012). A recent study showed that an immunestimulatory outer membrane protein of $A$. muciniphila (Amuc_1100) (Ottman et al. 2017b) is able to ameliorate the metabolic symptoms of obese and diabetic mice (Plovier et al. 2017). However, Amuc_1100 was not found to be differentially regulated in this study.

In addition, A. muciniphila upregulated several two-component systems in the co-cultures. Twocomponent systems consist of a membrane bound sensor histidine kinase and a cytoplasmic response regulator, which are often encoded by adjacent genes, enable bacteria to response to changing environment by altering gene expression (Monedero et al. 2017). However, the roles of two-component systems in $A$. muciniphila grown in the co-cultures were not yet identified. Studies showed that they could be involved in the regulation of physiological processes in commensal bacteria, such as stress responses, regulation of metabolism, and resistance to antimicrobial peptides (Monedero et al. 2017). The gastrointestinal pathogen, enterohemorrhagic E. coli (EHEC), was reported to encode the two-component system FusKR. This system provides a growth advantage and modulates the expression of virulence genes upon sensing of fucose liberated by Bacteroides thetaiotaomicron during growth in media containing mucin (Pacheco et al. 2012). The metabolism of mucin-derived fucose by A. muciniphila yielded 1,2-propanediol (Ottman et al. 2017a). As such, fucose metabolism by $A$. muciniphila could confer colonization resistance against the fucose-dependent enteric pathogens (Pickard and Chervonsky 2015).

In conclusion, we demonstrated the use of metatranscriptomics to provide in-depth mechanistic understanding of bacterial interaction. The trophic interaction between mucosal keystone species $A$. muciniphila and A. caccae could result in beneficial butyrate production at close proximity to the host epithelium. We revealed the expressional changes of A. muciniphila in response to A. caccae and demonstrated the provider role of A. muciniphila by upregulating the mucolytic activity to sustain the community at the mucosa niche. 


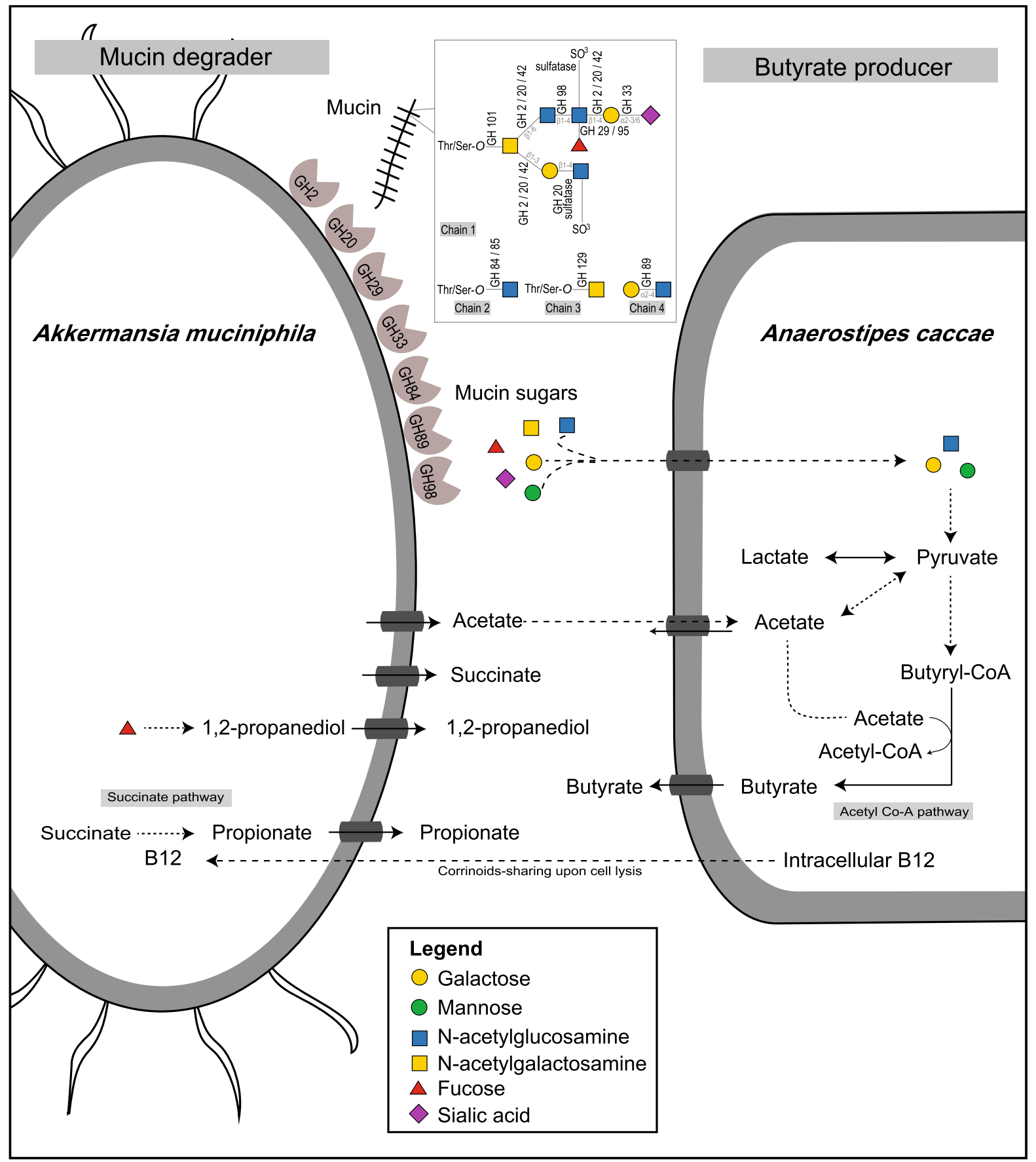

Fig. 4 Schematic representation of mucin-driven trophic interaction between A. muciniphila and A. caccae. A. muciniphila degrades oligosaccharides chain of mucin by extracellular glycosyl hydrolases. The structure for $O$-linked glycan chains and CAZymes action sites are adapted from Tailford et al. (2015). Chain 1 is a hypothetical mucin glycan chain, chain 2 is $O$-GlcNAc often found on other glycoproteins, chain 3 (Tn antigen) and chain 4 are found in gastro-duodenal mucin. In addition, mannose could be released from degradation of $\mathrm{N}$ linked glycan chains. A. caccae utilises some of the mucinderived sugars (galactose, mannose and GlcNAc) and acetate released by A. muciniphila for growth and concomitant butyrate production 
Acknowledgements We thank Thi Phuong Nam Bui, Kees C.H. van der Ark, Maria Suarez-Diez, Teunke van Rossum and Fons Stams for their constructive comments.Furthermore, we would like to thank Bart Nijsse for technical support. This work was partly supported by a special grant from Nutricia Research, the SIAM Gravitation Grant 024.002.002 of the Netherlands Organization for Scientific Research, and the IP/OP program Systems Biology (project KB-17-003.02-023).

Author contributions LWC, WMdV, JK and CB contributed to study conception. LWC, SA and $\mathrm{CB}$ contributed to experimental design. LWC performed the experiments. LWC and $\mathrm{BH}$ analysed data. LWC, BH, SA, PS, WMdV, JK and CB interpreted data and revised the manuscript. $\mathrm{LWC}$ and $\mathrm{BH}$ wrote the manuscript.

\section{Compliance with ethical standards}

Conflict of interest JK is the employee of Nutricia Research. LWC and CB are financially supported by Nutricia Research. There was no involvement of the company in the content of this work.

Open Access This article is distributed under the terms of the Creative Commons Attribution 4.0 International License (http:// creativecommons.org/licenses/by/4.0/), which permits unrestricted use, distribution, and reproduction in any medium, provided you give appropriate credit to the original author(s) and the source, provide a link to the Creative Commons license, and indicate if changes were made.

\section{References}

Afgan E et al (2016) The Galaxy platform for accessible, reproducible and collaborative biomedical analyses: 2016 update. Nucleic Acids Res 44:W3-W10. https://doi.org/10. 1093/nar/gkw343

Anders S, Pyl PT, Huber W (2015) HTSeq-a Python framework to work with high-throughput sequencing data. Bioinformatics 31:166-169

Anes J, McCusker MP, Fanning S, Martins M (2015) The ins and outs of RND efflux pumps in E coli. Front Microbiol 6:587. https://doi.org/10.3389/fmicb.2015.00587

Bailey TL et al (2009) MEME SUITE: tools for motif discovery and searching. Nucleic Acids Res 37:W202-208. https:// doi.org/10.1093/nar/gkp335

Belenguer A, Duncan SH, Calder AG, Holtrop G, Louis P, Lobley GE, Flint HJ (2006) Two routes of metabolic crossfeeding between Bifidobacterium adolescentis and butyrate-producing anaerobes from the human gut. Appl Environ Microbol 72:3593-3599. https://doi.org/10.1128/ Aem.72.5.3593-3599.2006

Belzer C, de Vos WM (2012) Microbes inside-from diversity to function: the case of Akkermansia. ISME J 6:1449-1458

Belzer C, Chia LW, Aalvink S, Chamlagain B, Piironen V, Knol J, de Vos WM (2017) Microbial metabolic networks at the mucus layer lead to diet-independent butyrate and vitamin
B12 production by intestinal symbionts. Mbio. https://doi. org/10.1128/mbio.00770-17

Caspi R et al (2014) The Metacyc database of metabolic pathways and enzymes and the BioCyc collection of Pathway/ Genome Databases. Nucleic Acids Res 42:D459-471. https://doi.org/10.1093/nar/gkt1103

Chomczynski P (1993) A reagent for the single-step simultaneous isolation of RNA, DNA and proteins from cell and tissue samples. BioTechniques 15(532-534):536-537

Collado MC, Derrien M, Isolauri E, de Vos WM, Salminen S (2007) Intestinal integrity and Akkermansia muciniphila, a mucin-degrading member of the intestinal microbiota present in infants, adults, and the elderly. Appl Environ Microbiol 73:7767-7770. https://doi.org/10.1128/AEM. 01477-07

de Vos WM (2017) Microbe Profile: akkermansia muciniphila: a conserved intestinal symbiont that acts as the gatekeeper of our mucosa. Microbiology. https://doi.org/10.1099/mic. 0.000444

De Vuyst L, Leroy F (2011) Cross-feeding between bifidobacteria and butyrate-producing colon bacteria explains bifdobacterial competitiveness, butyrate production, and gas production. Int J Food Microbiol 149:73-80. https://doi. org/10.1016/j.ijfoodmicro.2011.03.003

Degnan PH, Taga ME, Goodman AL (2014) Vitamin B12 as a modulator of gut microbial ecology. Cell Metab 20:769-778. https://doi.org/10.1016/j.cmet.2014.10.002

Derrien M, Vaughan EE, Plugge CM, de Vos WM (2004) Akkermansia muciniphila gen. nov., sp. nov., a human intestinal mucin-degrading bacterium. Int $\mathrm{J}$ Syst Evol Microbiol 54:1469-1476. https://doi.org/10.1099/ijs.0. 02873-0

Derrien M, Collado MC, Ben-Amor K, Salminen S, de Vos WM (2008) The mucin degrader Akkermansia muciniphila is an abundant resident of the human intestinal tract. Appl Environ Microb 74:1646-1648. https://doi.org/10.1128/ Aem.01226-07

Derrien M, Belzer C, de Vos WM (2016) Akkermansia muciniphila and its role in regulating host functions. Microbial Pathogenesis. https://doi.org/10.1016/j.micpath.2016.02. 005

Dimroth P, Schink B (1998) Energy conservation in the decarboxylation of dicarboxylic acids by fermenting bacteria. Arch Microbiol 170:69-77

Duncan SH, Louis P, Flint HJ (2004) Lactate-utilizing bacteria, isolated from human feces, that produce butyrate as a major fermentation product. Appl Environ Microbiol 70:5810-5817. https://doi.org/10.1128/AEM.70.10.58105817.2004

Everard A et al (2013) Cross-talk between Akkermansia muciniphila and intestinal epithelium controls diet-induced obesity. P Natl Acad Sci 110:9066-9071

Falony G, Vlachou A, Verbrugghe K, De Vuyst L (2006) Crossfeeding between Bifidobacterium longum BB536 and acetate-converting, butyrate-producing colon bacteria during growth on oligofructose. Appl Environ Microb 72:7835-7841

Fegatella F, Lim J, Kjelleberg S, Cavicchioli R (1998) Implications of rRNA operon copy number and ribosome content in the marine oligotrophic ultramicrobacterium 
Sphingomonas sp. strain RB2256. Appl Environ Microbiol 64:4433-4438

Galdiero S, Falanga A, Cantisani M, Tarallo R, Della Pepa ME, D'Oriano V, Galdiero M (2012) Microbe-host interactions: structure and role of Gram-negative bacterial porins. Curr Protein Peptide Sci 13:843-854

Grander C et al (2017) Recovery of ethanol-induced Akkermansia muciniphila depletion ameliorates alcoholic liver disease. Gut. https://doi.org/10.1136/gutjnl-2016-313432

Haas BJ, Chin M, Nusbaum C, Birren BW, Livny J (2012) How deep is deep enough for RNA-Seq profiling of bacterial transcriptomes? Bmc Genomics. https://doi.org/10.1186/ 1471-2164-13-734

Huson DH, Mitra S, Ruscheweyh HJ, Weber N, Schuster SC (2011) Integrative analysis of environmental sequences using MEGAN4. Genome Res 21:1552-1560. https://doi. org/10.1101/gr.120618.111

Joshi N, Fass J (2011) Sickle: A sliding-window, adaptive, quality-based trimming tool for FastQ files (Version 1.33) [Software]

Koh A, De Vadder F, Kovatcheva-Datchary P, Backhed F (2016) From dietary fiber to host physiology: short-chain fatty acids as key bacterial metabolites. Cell 165:1332-1345. https://doi.org/10.1016/j.cell.2016.05.041

Kopylova E, Noe L, Touzet H (2012) SortMeRNA: fast and accurate filtering of ribosomal RNAs in metatranscriptomic data. Bioinformatics 28:3211-3217

Koropatkin NM, Cameron EA, Martens EC (2012) How glycan metabolism shapes the human gut microbiota. Nat Rev Microbiol 10:323-335. https://doi.org/10.1038/ Nrmicro2746

Kostic AD, Xavier RJ, Gevers D (2014) The microbiome in inflammatory bowel disease: current status and the future ahead. Gastroenterology 146:1489-1499. https://doi.org/ 10.1053/j.gastro.2014.02.009

Krogh A, Larsson B, von Heijne G, Sonnhammer EL (2001) Predicting transmembrane protein topology with a hidden Markov model: application to complete genomes. J Mol Biol 305:567-580. https://doi.org/10.1006/jmbi.2000. 4315

Langmead B, Salzberg SL (2012) Fast gapped-read alignment with Bowtie 2. Nat Methods 9:357-359. https://doi.org/10. 1038/nmeth.1923

Lopez-Siles M, Khan TM, Duncan SH, Harmsen HJM, GarciaGil LJ, Flint HJ (2012) Cultured representatives of two major phylogroups of human colonic faecalibacterium prausnitzii can utilize pectin, uronic acids, and hostderived substrates for growth. Appl Environ Microb 78:420-428. https://doi.org/10.1128/Aem.06858-11

Lopez-Siles M et al (2014) Mucosa-associated faecalibacterium prausnitzii and escherichia coli co-abundance can distinguish irritable bowel syndrome and inflammatory bowel disease phenotypes. Int J Med Microbiol 304:464-475. https://doi.org/10.1016/j.ijmm.2014.02.009

Louis P, Flint HJ (2009) Diversity, metabolism and microbial ecology of butyrate-producing bacteria from the human large intestine. FEMS Microbiol Lett 294:1-8. https://doi. org/10.1111/j.1574-6968.2009.01514.x

Louis P, Flint HJ (2017) Formation of propionate and butyrate by the human colonic microbiota. Environ Microbiol 19:29-41. https://doi.org/10.1111/1462-2920.13589
Love M, Anders S, Kim V, Huber W (2015) RNA-Seq workflow: gene-level exploratory analysis and differential expression [version 1; referees: 2 approved] vol 4.

Magnusdottir S, Ravcheev D, de Crecy-Lagard V, Thiele I (2015) Systematic genome assessment of B-vitamin biosynthesis suggests co-operation among gut microbes. Front Genet 6:148. https://doi.org/10.3389/fgene.2015. 00148

Marcobal A, Southwick AM, Earle KA, Sonnenburg JL (2013) A refined palate: bacterial consumption of host glycans in the gut. Glycobiology 23:1038-1046. https://doi.org/10. 1093/glycob/cwt040

Martin M (2011) Cutadapt removes adapter sequences from high-throughput sequencing reads. EMBnet J. https://doi. org/10.14806/ej.17.1.200

Miller RS, Hoskins LC (1981) Mucin degradation in human colon ecosystems. fecal population densities of mucindegrading bacteria estimated by a "most probable number" method. Gastroenterology 81:759-765

Monedero V, Revilla-Guarinos A, Zúñiga M (2017) Physiological role of two-component signal transduction systems in food-associated lactic acid bacteria. Advances Appl Microbiol 99:1-51

Moran AP, Gupta A, Joshi L (2011) Sweet-talk: role of host glycosylation in bacterial pathogenesis of the gastrointestinal tract. Gut 60:1412-1425

Nava GM, Friedrichsen HJ, Stappenbeck TS (2011) Spatial organization of intestinal microbiota in the mouse ascending colon. ISME J 5:627-638. https://doi.org/10. 1038/ismej.2010.161

Nikaido H, Takatsuka Y (2009) Mechanisms of RND multidrug efflux pumps. Biochim Biophys Acta 1794:769-781. https://doi.org/10.1016/j.bbapap.2008.10.004

Ottman $\mathrm{N}$ et al (2016) Characterization of outer membrane proteome of akkermansia muciniphila reveals sets of novel proteins exposed to the human intestine. Front Microbiol 7:1157. https://doi.org/10.3389/fmicb.2016.01157

Ottman N et al (2017a) Genome-scale model and omics analysis of metabolic capacities of Akkermansia muciniphila reveal a preferential mucin-degrading lifestyle. Appl Environ Microbiol. https://doi.org/10.1128/AEM.01014-17

Ottman N et al (2017b) Pili-like proteins of Akkermansia muciniphila modulate host immune responses and gut barrier function. PLoS ONE 12:e0173004. https://doi.org/ 10.1371/journal.pone.0173004

Ouwehand AC, Derrien M, de Vos W, Tiihonen K, Rautonen N (2005) Prebiotics and other microbial substrates for gut functionality. Curr Opin Biotechnol 16:212-217. https:// doi.org/10.1016/j.copbio.2005.01.007

Ouwerkerk JP, de Vos WM, Belzer C (2013) Glycobiome: bacteria and mucus at the epithelial interface. Best Pract Res Clin Gastroenterol 27:25-38. https://doi.org/10.1016/ j.bpg.2013.03.001S1521-6918(13)00038-3

Ouwerkerk JP, van der Ark KC, Davids M, Claassens NJ, Robert Finestra T, de Vos WM, Belzer C (2016) Adaptation of Akkermansia muciniphila to the oxic-anoxic interface of the mucus layer. Appl Environ Microbiol. https://doi.org/ 10.1128/AEM.01641-16

Pacheco AR, Curtis MM, Ritchie JM, Munera D, Waldor MK, Moreira CG, Sperandio V (2012) Fucose sensing regulates 
bacterial intestinal colonization. Nature 492:113-117. https://doi.org/10.1038/nature11623

Petersen TN, Brunak S, von Heijne G, Nielsen H (2011) SignalP 4.0: discriminating signal peptides from transmembrane regions. Nat Methods 8:785-786. https://doi.org/10.1038/ nmeth. 1701

Pickard JM, Chervonsky AV (2015) Intestinal Fucose as a Mediator of Host-Microbe Symbiosis. J Immunol 194:5588-5593. https://doi.org/10.4049/jimmunol. 1500395

Plichta DR et al (2016) Transcriptional interactions suggest niche segregation among microorganisms in the human gut. Nat Microbiol. https://doi.org/10.1038/Nmicrobiol. 2016.152

Plovier $\mathrm{H}$ et al (2017) A purified membrane protein from Akkermansia muciniphila or the pasteurized bacterium improves metabolism in obese and diabetic mice. Nat Med 23:107-113. https://doi.org/10.1038/nm.4236

Plugge CM (2005) Anoxic media design, preparation, and considerations. Methods Enzymol 397:3-16. https://doi. org/10.1016/S0076-6879(05)97001-8

Png CW et al (2010) Mucolytic bacteria with increased prevalence in IBD mucosa augment in vitro utilization of mucin by other bacteria. Am J Gastroenterol 105:2420-2428. https://doi.org/10.1038/ajg.2010.281

Rios-Covian D, Gueimonde M, Duncan SH, Flint HJ, de los Reyes-Gavilan CG (2015) Enhanced butyrate formation by cross-feeding between Faecalibacterium prausnitzii and Bifidobacterium adolescentis. FEMS Microbiol Lett. https://doi.org/10.1093/femsle/fnv176

Riviere A, Gagnon M, Weckx S, Roy D, De Vuyst L (2015) Mutual Cross-Feeding Interactions between Bifidobacterium longum subsp longum NCC2705 and Eubacterium rectale ATCC 33656 explain the bifidogenic and butyrogenic effects of arabinoxylan oligosaccharides. Appl Environ Microb 81:7767-7781

Schluter J, Foster KR (2012) The evolution of mutualism in gut microbiota via host epithelial selection. PLoS Biol 10:e1001424. https://doi.org/10.1371/journal.pbio.1001424

Schurch NJ et al (2016) How many biological replicates are needed in an RNA-seq experiment and which differential expression tool should you use? RNA 22:839. https://doi. org/10.1261/rna.058339.116

Schwab C, Ruscheweyh HJ, Bunesova V, Pham VT, Beerenwinkel N, Lacroix C (2017) Trophic interactions of infant bifidobacteria and eubacterium hallii during 1-fucose and fucosyllactose degradation. Front Microbiol 8:95. https:// doi.org/10.3389/fmicb.2017.00095

Schwiertz A et al (2002) Anaerostipes caccae gen. nov., sp. nov., a new saccharolytic, acetate-utilising, butyrate-producing bacterium from human faeces. Syst Appl Microbiol 25:46-51. https://doi.org/10.1078/0723-2020-00096

Shetty SA, Hugenholtz F, Lahti L, Smidt H, Vos WMd (2016) Intestinal microbiome landscaping: insight in community assemblage and implications for microbial modulation strategies FEMS Microbiology Reviews

Swidsinski A et al (2011) Acute appendicitis is characterised by local invasion with Fusobacterium nucleatum/necrophorum. Gut 60:34-40. https://doi.org/10.1136/gut.2009. 191320

Tailford LE, Crost EH, Kavanaugh D, Juge N (2015) Mucin glycan foraging in the human gut microbiome. Front Genet 6:81. https://doi.org/10.3389/fgene.2015.00081

Van den Abbeele P et al (2013) Butyrate-producing Clostridium cluster XIVa species specifically colonize mucins in an in vitro gut model. ISME J 7:949-961. https://doi.org/10. 1038/ismej.2012.158ismej2012158

van der Walt S, Colbert SC, Varoquaux G (2011) The numpy array: a structure for efficient numerical computation. Comput Sci Eng 13:22-30

van Passel MWJ et al (2011) The genome of akkermansia muciniphila, a dedicated intestinal mucin degrader, and its use in exploring intestinal metagenomes. PLoS ONE. https://doi.org/10.1371/journal.pone.0016876

Veiga P et al (2010) Bifidobacterium animalis subsp. lactis fermented milk product reduces inflammation by altering a niche for colitogenic microbes. Proc Natl Acad Sci 107:18132-18137. https://doi.org/10.1073/pnas. 1011737107

Vital M, Howe AC, Tiedje JM (2014) Revealing the bacterial butyrate synthesis pathways by analyzing (meta)genomic data. MBio. https://doi.org/10.1128/mbio.00889-14

Yin Y, Mao X, Yang J, Chen X, Mao F, Xu Y (2012) dbCAN: a web resource for automated carbohydrate-active enzyme annotation. Nucl Acids Res 40:W445-451. https://doi.org/ 10.1093/nar/gks479

Zoetendal EG, Booijink CC, Klaassens ES, Heilig HG, Kleerebezem M, Smidt H, de Vos WM (2006) Isolation of RNA from bacterial samples of the human gastrointestinal tract. Nat Protoc 1:954-959. https://doi.org/10.1038/nprot. 2006.143 